\title{
A COMBINATORIAL IDENTITY AND ITS APPLICATION TO GAUSSIAN MEASURES
}

\author{
LUIGI ACCARDI \\ Centro Vito Volterra \\ Facoltà di Economia \\ Università di Roma "Tor Vergata" \\ 00133 Roma, Italy \\ E-mail: accardi@volterra.mat.uniroma2.it \\ HUI-HSIUNG KUO \\ Department of Mathematics \\ Louisiana State University \\ Baton Rouge, LA 70803, U.S.A. \\ E-mail: kuo@math.lsu.edu \\ AUREL I. STAN \\ Department of Mathematics \\ The Ohio State University at Marion \\ 1465 Mount Vernon Avenue \\ Marion, $O H$ 43302, U.S.A. \\ E-mail: stan.7@osu.edu
}

2000 Mathematics Subject Classifications: 05E35, 60H40.

Key words and phrases: commutator, annihilation operator, creation operator, neutral (preservation) operator.

Assuming that a probability measure on $\mathbb{R}^{d}$ has finite moments of any order, its moments are completely determined by two family of operators. The first family is composed of the neutral (preservation) operators. The second family consists of the commutators between the annihilation and creation operators. As a confirmation of this fact, a characterization of the Gaussian probability measures in terms of these two families of operators is given. The proof of this characterization relies on a simple combinatorial identity. 


\section{Introduction}

Let $d$ be a positive integer and $\mu$ a probability measure on the Borel subsets of $\mathbb{R}^{d}$. We assume that $\mu$ has finite moments of any order, (i.e., $\int_{\mathbb{R}^{d}}\left|x_{i}\right|^{p} d x<\infty$, for all $p>0$ and $i \in\{1,2, \ldots, d\}$, where $x_{i}$, denotes the $i$-th coordinate of a generic vector $x=\left(x_{1}, x_{2}, \ldots, x_{d}\right)$ in $\left.\mathbb{R}^{d}\right)$. For all non-negative integers $n$, we consider the space $F_{n}$ of all polynomial functions $f\left(x_{1}, x_{2}, \ldots, x_{d}\right)$, of $d$-variables, such that $\operatorname{deg}(f) \leq n$, where $\operatorname{deg}(f)$ denotes the total degree of $f$. Because $\mu$ has finite moments of any order, it follows from Hölder's inequality that $F_{n} \subset L^{2}\left(\mathbb{R}^{d}, \mu\right)$, for all $n \geq 0$. Moreover, $F_{n}$ is a closed subspace of $L^{2}\left(\mathbb{R}^{d}, \mu\right)$, since $F_{n}$ is finite dimensional, for all $n \geq 0$. Thus, for all $n \geq 0$, we may define the spaces $G_{n}:=F_{n} \ominus F_{n-1}$, where $F_{n} \ominus F_{n-1}$ denotes the orthogonal complement of $F_{n-1}$ into $F_{n}$ with respect to the inner product given by $\mu$, and $F_{-1}:=\{0\}$ is the null space. We define the Hilbert space $\mathcal{H}$ as the direct sum of the orthogonal subspaces $\left\{G_{n}\right\}_{n \geq 0}$ (i.e., $\left.\mathcal{H}:=\oplus_{n \geq 0} G_{n}\right)$. Let $V$ be the space of all polynomial functions of $d$-variables: $x_{1}, x_{2}, \ldots, x_{d}$, and of arbitrary degree, in which any two polynomials, that are equal $\mu$-almost surely, are considered to be identical.

For all $i \in\{1,2, \ldots, d\}$, we denote by $X_{i}$ the operator of multiplication by the variable $x_{i}$. This operator is defined on the space $V$, which is dense in $\mathcal{H}$, as $\left(X_{i} f\right)\left(x_{1}, x_{2}, \ldots, x_{d}\right):=x_{i} f\left(x_{1}, x_{2}, \ldots, x_{d}\right)$.

The following lemma leads us to the notion of creation, neutral (preservation), and annihilation operators. See [2] for details.

Lernma 1.1. For all $n \geq 0$ and $i \in\{1,2, \ldots, d\}, X_{i} G_{n} \perp G_{k}$, for all $k \neq$ $n-1, n, n+1$, where $\perp$ denotes the orthogonality relation with respect to the inner product generated by the probability measure $\mu$.

From Lemma 1.1, it follows that, for all $n \geq 0$ and $i \in\{1,2, \ldots, d\}, X_{i} G_{n} \subset$ $G_{n-1} \oplus G_{n} \oplus G_{n+1}$. This means that, for all $f \in G_{n}, X_{i} f=f_{i, n-1}+f_{i, n}+$ $f_{i, n+1}$, for some $f_{i, n-1} \in G_{n-1}, f_{i, n} \in G_{n}$, and $f_{i, n+1} \in G_{n+1}$. We define three family of operators $D_{n}^{+}(i): G_{n} \rightarrow G_{n+1}, D_{n}^{+}(i) f:=f_{i, n+1}, D_{n}^{0}(i)$ : $G_{n} \rightarrow G_{n}, D_{n}^{0}(i) f:=f_{i, n}$, and $D_{n}^{-}(i): G_{n} \rightarrow G_{n-1}, D_{n}^{-}(i) f:=f_{i, n-1}$, and observe that the restriction of the multiplication operator $X_{i}$ to the space $G_{n}, X_{i} \mid G_{n}$, satisfies the relation:

$$
X_{i} \mid G_{n}=D_{n}^{+}(i)+D_{n}^{0}(i)+D_{n}^{-}(i),
$$

for all $n \geq 0$ and $i \in\{1,2, \ldots, d\}$. 
We extend now this family of operators, by linearity, to the whole space $V$ of polynomial functions. That means, if $\varphi=\sum_{n \geq 0} f_{n}$, where for each $n \geq 0, f_{n} \in G_{n}$, and only a finite number of the terms $f_{n}$ are not zero, then we define, for all $n \geq 0$ and $i \in\{1,2, \ldots, d\}$,

$$
\begin{aligned}
a^{+}(i) \varphi & :=\sum_{n=0}^{\infty} D_{n}^{+}(i) f_{n} \\
a^{0}(i) \varphi & :=\sum_{n=0}^{\infty} D_{n}^{0}(i) f_{n}
\end{aligned}
$$

and

$$
a^{-}(i) \varphi:=\sum_{n=0}^{\infty} D_{n}^{-}(i) f_{n} .
$$

Equality (1) becomes now the following fundamental relation of this theory:

$$
X_{i}=a^{+}(i)+a^{0}(i)+a^{-}(i)
$$

for all $i \in\{1,2, \ldots, d\} . a^{+}(i)$ is called a creation operator, $a^{0}(i)$ a neutral or preservation operator, and $a^{-}(i)$ an annihilation operator, for all $i \in\{1,2, \ldots, d\}$.

A probability measure $\mu$ on $\mathbb{R}^{d}$, having finite moments of any order, is called polynomially factorisable, if for any non-negative integers $i_{1}, i_{2}, \ldots$, $i_{d}, E\left[x_{1}^{i_{1}} x_{2}^{i_{2}} \cdots x_{d}^{i_{d}}\right]=E\left[x_{1}^{i_{1}}\right] E\left[x_{2}^{i_{2}}\right] \cdots E\left[x_{d}^{i_{d}}\right]$. A probability measure $\mu$ that is a product of $d$ probability measures $\mu_{1}, \mu_{2}, \ldots, \mu_{d}$, on $\mathbb{R}$, each of them having finite moments of any order, is clearly polynomially factorisable by Fubini's theorem, but the converse is not true and this was shown by a counterexample in [2]. If, for each $i \in\{1,2, \ldots, d\}$, we regard $X_{i}$ as the random variable that associates to each outcome $x=\left(x_{1}, x_{2}, \ldots, x_{d}\right)$, from our sample space, $\mathbb{R}^{d}$, its $i$-th coordinate $x_{i}$, then the notion of polynomially factorisability can be understood as a weak form of independence of the random variables $X_{1}, X_{2}, \ldots, X_{d}$. If one is dissatisfied with the fact that our sample space is the particular one $\mathbb{R}^{d}$, and would prefer to have a general theory for an arbitrary sample space $\Omega$ and arbitrary random variables $Y_{1}, Y_{2}, \ldots, Y_{d}$ defined on $\Omega$, having finite moments of any order, then he(she) can take $\mu$ to be the joint probability distribution of these random variables and in this way the whole theory is moved on $\mathbb{R}^{d}$. The random variables $Y_{1}, Y_{2}, \ldots, Y_{d}$ defined on $\Omega$, are thus replaced by the coordinate 
random variables $X_{1}, X_{2}, \ldots, X_{d}$ on $\mathbb{R}^{d}$. Therefore, one can see that by working only on $\mathbb{R}^{d}$ we do not loose anything from the generality of this theory.

The following theorem was proved in [2]:

Theorem 1.1. A probability measure $\mu$ on $\mathbb{R}^{d}$, having finite moments of any order, is polynomially factorisable, if and only if for any $j, k \in$ $\{1,2, \ldots, d\}$, such that $j \neq k$, any operator from the set $\left\{a^{-}(j), a^{0}(j)\right.$, $\left.a^{+}(j)\right\}$ commutes with any operator from the set $\left\{a^{-}(k), a^{0}(k), a^{+}(k)\right\}$.

\section{A Combinatorial Identity}

We will prove now an identity that will have remarkable connections with the standard Gaussian probability measure on $\mathbb{R}$.

Lemma 2.1. For all natural numbers $n$, we have:

$$
\sum_{1 \leq j_{1}<j_{2}<\cdots<j_{n} \leq 2 n}\left(j_{1}-1\right)^{+}\left(j_{2}-3\right)^{+} \ldots\left(j_{n}-(2 n-1)\right)^{+}=(2 n-1) ! !
$$

where $a^{+}:=\max (a, 0)$, for all real numbers $a$.

Proof. Let us consider $2 n$ people called: $a_{1}, a_{2}, \ldots, a_{2 n}$, such that any two of them are of different ages. We assume that $a_{1}<a_{2}<\cdots<a_{2 n}$, where $a_{i}<a_{j}$ means that person $a_{i}$ is younger than person $a_{j}$. We would like to split these $2 n$ people into $n$ disjoint teams (couples) of two people each. Each couple will play a game of tennis with any other couple. We can count the number of such partitions in two different ways.

The first way is to use the multinomial coefficient $\left(\begin{array}{c}2 n \\ 2,2, \ldots, 2\end{array}\right)$, to count the number of ways in which we can form team 1 , team $2, \ldots$, team $n$. After fixing team 1 , team $2, \ldots$, team $n$, if for example we move the two people from team 1 into team 2 , and the two people from team 2 into team 1 , leaving all the other teams unchanged, then from the playing tennis point of view the teams are the same, they have only changed their names (team 2 is called now team 1 , and team 1 is team 2 , but the players within each team have remained the same). Thus by permuting the names (counting) of the teams we over-counted the number of partitions $n$ ! times. Hence, the total number of partitions is:

$$
\frac{\left(\begin{array}{c}
2 n \\
2,2, \ldots, 2
\end{array}\right)}{n !}=\frac{\frac{(2 n) !}{2 ! 2 ! \cdots 2 !}}{n !}=\frac{(2 n) !}{2^{n} n !}=(2 n-1) ! !
$$


Therefore, we have obtained the number from the right-hand side of the formula from our lemma.

The second way is to order first the two people within each team and then to order the teams. Since the team $\left(a_{i}, a_{j}\right)$ is the same as the team $\left(a_{j}, a_{i}\right)$, to avoid over-counting, we will always specify a team by listing first the younger member of the team and second the older member of the team. Thus for example $\left(a_{1}, a_{2}\right)$ is a legitimate team, while $\left(a_{2}, a_{1}\right)$ is not. We also order the teams among themselves, by saying that team 1 is less than team 2 if the older person from team 1 is younger than the older person from team 2. To avoid over-counting we will select the teams in their increasing order (that means the older member of team 1 is younger than the older member of team 2 who in turn is younger than the older member of team 3 and so on). We count now the number of partitions, by selecting first the older member from each team in their increasing order. Let $a_{j_{1}}$ be the older member from team $1, a_{j_{2}}$ the older member from team 2 , and so on. Since we have ordered the teams, we have $1 \leq j_{1}<j_{2}<\cdots<j_{n} \leq 2 n$. Let us fix for the moment $j_{1}, j_{2}, \ldots, j_{n}$. We need to select now the younger member from each team, whom we call $a_{i_{1}}, a_{i_{2}}, \ldots, a_{i_{m}}$. Since the younger person from team $1, a_{i_{1}}$, is younger than his(her) team-mate $a_{j_{1}}$, we must have $1 \leq i_{1}<j_{1}$. Therefore, there are $j_{1}-1$ choices for $i_{1}$, namely: 1 , $2, \ldots, j_{1}-1$, if $j_{1}>1$, and no choice (room) if $j_{1}=1$. Thus, we can say that in both cases, the number of ways to select a younger partner for person $a_{j_{1}}$ is $\left(j_{1}-1\right)^{+}$. Let us assume now that the partner $a_{i_{1}}$ of $a_{j_{1}}$ has been chosen and fixed and let us proceed to select the partner $a_{i_{2}}$ of $a_{j_{2}}$ from team 2. Since $a_{i_{2}}$ is younger than $a_{j_{2}}$, we must have $i_{2}<j_{2}$. Since there are $j_{2}-1$ numbers (positions) less than $j_{2}$, namely: $1,2, \ldots, j_{2}-1$, out of which two are already occupied by $i_{1}$ and $j_{1}$, we can see that there are $j_{2}-3$ choices left for $i_{2}$, if $j_{2}>3$, and no choices if $j_{2} \leq 3$. Thus the number of ways in which $i_{2}$ can be selected is $\left(j_{2}-3\right)^{+}$. Similarly we can see that after $i_{1}$ and $i_{2}$ have been selected and fixed, the number of ways in which we can select a younger partner for the player $a_{j_{3}}$ is $\left(j_{3}-5\right)^{+}$, and so on. Finally, there are $\left(j_{n}-(2 n-1)\right)^{+}$ways to select a younger partner for the player $a_{j_{n}}$. Using the generalized principle of counting, we conclude that if the older players from each team: $a_{j_{1}}, a_{j_{2}}, \ldots, a_{j_{n}}$ have been fixed and ordered as $j_{1}<j_{2}<\cdots<j_{n}$, then the number of ways in which we can pair each of them with a younger partner is: $\left(j_{1}-1\right)^{+}\left(j_{2}-3\right)^{+} \ldots\left(j_{n}-(2 n-1)\right)^{+}$. Thus the total number of partitions is: $\sum_{1 \leq j_{1}<j_{2}<\cdots<j_{n} \leq 2 n}\left(j_{1}-1\right)^{+}\left(j_{2}-3\right)^{+} \cdots\left(j_{n}-(2 n-1)\right)^{+}$and so, our identity is proved. 


\section{Standard Gaussian Probability Measure}

In this section we characterize the standard Gaussian probability measure on $\mathbb{R}^{d}$ in terms of the neutral (preservation) and commutators between the creation and annihilation operators.

Theorem 3.1. The standard Gaussian probability measure on $\mathbb{R}^{d}$, i.e., the probability measure given by the density function

$$
f\left(x_{1}, x_{2}, \ldots, x_{d}\right)=(2 \pi)^{-\frac{d}{2}} e^{-\frac{x_{1}^{2}+x_{2}^{2}+\cdots+x_{d}^{2}}{2}},
$$

is the only probability measure, on $\mathbb{R}^{d}$, having finite moments of all orders, such that for $i, j, k \in\{1,2, \ldots, d\}$,

$$
a^{0}(i)=0
$$

and

$$
\left[a^{-}(j), a^{+}(k)\right]=\delta_{j, k} I,
$$

where $\left[a^{-}(j), a^{+}(k)\right]:=a^{-}(j) a^{+}(k)-a^{+}(k) a^{-}(j)$ denotes the commutator of $a^{-}(j)$ and $a^{+}(k)$, and $\delta_{j, k}=1$, if $j=k$, and $\delta_{j, k}=0$, if $j \neq k$, is the Kronecker symbol. Here I denotes the identity operator of the space $V$ of all polynomial functions of $d$ real variables: $x_{1}, x_{2}, \ldots, x_{d}$. In equalities (6) and (7), the domain of $a^{0}(i), a^{-}(j)$, and $a^{+}(k)$ is considered to be $V$.

Proof. $(\Leftrightarrow)$ If $\mu$ is the standard Gaussian probability measure on $\mathbb{R}^{d}$, then it is well-known that relations (6) and (7) hold.

$\Leftrightarrow$ Let us assume now that $\mu$ is a probability measure on $\mathbb{R}^{d}$, having finite moments of all orders, such that for $i, j, k \in\{1,2, \ldots, d\}, a^{0}(i)=0$ and $\left[a^{-}(j), a^{+}(k)\right]=\delta_{j, k} I$. We will compute all the mixed moments of $\mu$. Since for all $j \neq k$, any operator from the set $\left\{a^{-}(j), a^{0}(j), a^{+}(j)\right\}$ commutes with any operator from the set $\left\{a^{-}(k), a^{0}(k), a^{+}(k)\right\}$, using Theorem 1.1 , we conclude that $\mu$ is polynomially factorisable. This means that, for all monomials $x_{1}^{i_{1}} x_{2}^{i_{2}} \cdots x_{d}^{i_{d}}$, we have

$$
E\left[x_{1}^{i_{1}} x_{2}^{i_{2}} \cdots x_{d}^{i_{d}}\right]=E\left[x_{1}^{i_{1}}\right] E\left[x_{2}^{i_{2}}\right] \cdots E\left[x_{d}^{i_{d}}\right],
$$

where $E$ denotes the expectation with respect to $\mu$.

Since the mixed moments of $\mu$ are the product of their corresponding marginal moments, it is enough to focus on computing the following moments: $E\left[x_{i}\right], E\left[x_{i}^{2}\right], E\left[x_{i}^{3}\right], \ldots$, where $i \in\{1,2, \ldots, d\}$ is an arbitrary fixed sub-script. Let $i \in\{1,2, \ldots, d\}$ and $m \in \mathbb{N}$ be fixed. Let $\phi:=1$, be the constant polynomial 1 . We call $\phi$ the vacuum vector. To obtain the 
monomial $x_{i}^{m}$, from the constant polynomial 1, we must apply the multiplication operator $X_{i}$, to the vacuum vector $\phi$, repeatedly $m$-times. That is, $x_{i}^{m}=X_{i} X_{i} \cdots X_{i} \phi=X_{i}^{m} \phi$. Since $X_{i}=a^{-}(i)+a^{0}(i)+a^{+}(i)$ and $a^{0}(i)=0$, we have $X_{i}=a^{-}(i)+a^{+}(i)$. Because the index $i$ has been fixed, we will denote $a^{-}(i)$ and $a^{+}(i)$ shortly by $a^{-}$and $a^{+}$, respectively. We denote by $\langle\cdot, \cdot\rangle$, the inner product with respect to $\mu$. Then, we have:

$$
\begin{aligned}
E\left[x_{i}^{m}\right] & =\left\langle x_{i}^{m}, 1\right\rangle \\
& =\left\langle X_{i}^{m} \phi, \phi\right\rangle \\
& =\left\langle\left(a^{-}+a^{+}\right)^{m} \phi, \phi\right\rangle \\
& =\left\langle\left(a^{-}+a^{+}\right)\left(a^{-}+a^{+}\right) \cdots\left(a^{-}+a^{+}\right) \phi, \phi\right\rangle \\
& =\sum_{\epsilon_{1}, \epsilon_{2}, \ldots, \epsilon_{m}}\left\langle a^{\epsilon_{1}} a^{\epsilon_{2}} \cdots a^{\epsilon_{m}} \phi, \phi\right\rangle,
\end{aligned}
$$

where $\epsilon_{j} \in\{-,+\}$, for all $j \in\{1,2, \ldots, m\}$. Actually, it will be better to count the epsilons from right to left since otherwise $\epsilon_{m}$ is applied first to the vacuum vector $\phi$, then $\epsilon_{m-1}$ and so on. Therefore, we have:

$$
E\left[x_{i}^{m}\right]=\sum_{\epsilon_{1}, \epsilon_{2}, \ldots, \epsilon_{m}}\left\langle a^{\epsilon_{m}} \cdots a^{\epsilon_{2}} a^{\epsilon_{1}} \phi, \phi\right\rangle .
$$

Let $j \in\{1,2, \ldots, m\}$. If $\epsilon_{j}=-$, then there exists $k_{j} \geq 0$, such that $a^{\epsilon_{j}}: G_{k_{j}} \rightarrow G_{k_{j}-1}$, and for this reason we call $a^{\epsilon_{j}}$ a backward step. Here, if $k_{j}=0$, then $G_{k_{j}-1}=G_{-1}:=\{0\}$ is the null space. If $\epsilon_{j}=+$, then there exists $k_{j} \geq 0$, such that $a^{\epsilon_{j}}: G_{k_{j}} \rightarrow G_{k_{j}+1}$, and for this reason we call $a^{\epsilon_{j}}$ a forward step.

If $m=2 n+1$ is odd, where $n$ is some non-negative integer, then in every term $\left\langle a^{\epsilon_{m}} \cdots a^{\epsilon_{2}} a^{\epsilon_{1}} \phi, \phi\right\rangle$, of the sum from formula (8), the number of forward steps is different from the number of backward steps. Since we start from $G_{0}$ (because $\phi \in G_{0}$ ) and we do a number of forward steps and a different number of backward steps, it is clear that either $a^{\epsilon_{m}} \cdots a^{\epsilon_{2}} a^{\epsilon_{1}} \phi=$ 0 , if the number of backward steps is greater than the number of forward steps, or $a^{\epsilon_{m}} \cdots a^{\epsilon_{2}} a^{\epsilon_{1}} \phi \in G_{k}$, for some $k \geq 1$ ( $k$ is actually odd, but this is not important for us), if the number of forward steps is greater than the number of backward steps. Because $G_{k}$ is orthogonal to $G_{0}$, for all $k \geq 1$, and $\phi \in G_{0}$ (here we refer to the $\phi$ that appears after the comma in $\left.\left\langle a^{\epsilon_{m}} \cdots a^{\epsilon_{2}} a^{\epsilon_{1}} \phi, \phi\right\rangle\right)$, we conclude that in both cases $\left\langle a^{\epsilon_{m}} \cdots a^{\epsilon_{2}} a^{\epsilon_{1}} \phi, \phi\right\rangle=0$, for all choices of $\epsilon_{1}, \epsilon_{2}, \ldots, \epsilon_{m}$. Thus the sum from formula (8), is a sum of zeros, and so $E\left[x^{m}\right]=0$, for all odd positive integers $m$.

If $m=2 n$ is even, where $n$ is a positive integer, then the only non-zero terms from the sum $\sum_{\epsilon_{1}, \epsilon_{2}, \ldots, \epsilon_{2 n}}\left\langle a^{\epsilon_{2 n}} \cdots a^{\epsilon_{2}} a^{\epsilon_{1}} \phi, \phi\right\rangle$, are eventually those in 
which the number of forward steps is equal to the number of backward steps. For these terms, since $a^{\epsilon_{m}} \cdots a^{\epsilon_{2}} a^{\epsilon_{1}} \phi \in G_{0}$, we can see that $a^{\epsilon_{2 n}} \cdots a^{\epsilon_{2}} a^{\epsilon_{1}} \phi$ is a constant polynomial, therefore a number, and so $\left\langle a^{\epsilon_{2 n}} \cdots a^{\epsilon_{2}} a^{\epsilon_{1}} \phi, \phi\right\rangle=$ $a^{\epsilon_{2 n}} \cdots a^{\epsilon_{2}} a^{\epsilon_{1}} \phi$. Since exactly $n$ of $\epsilon_{1}, \epsilon_{2}, \ldots, \epsilon_{2 n}$, are equal to - (the other $n$ being equal to + ), let us call the positions of these minus-epsilons by $j_{1}, j_{2}, \ldots, j_{n}$, where $j_{1}<j_{2}<\cdots<j_{n}$. That means $\epsilon_{j}=-$, if $j \in\left\{j_{1}, j_{2}, \ldots, j_{n}\right\}$, and $\epsilon_{j}=+$, if $j \in\{1,2, \ldots, 2 n\} \backslash\left\{j_{1}, j_{2}, \ldots, j_{n}\right\}$. Formula (8) becomes now:

$$
\begin{aligned}
& E\left[x_{i}^{m}\right]=\sum_{\epsilon_{1}, \epsilon_{2}, \ldots, \epsilon_{m}}\left\langle a^{\epsilon_{m}} \cdots a^{\epsilon_{2}} a^{\epsilon_{1}} \phi, \phi\right\rangle \\
&=\sum_{1 \leq j_{1}<j_{2}<\cdots<j_{n} \leq 2 n}\left(a^{+}\right)^{2 n-j_{n}} a^{-}\left(a^{+}\right)^{j_{n}-1-j_{n-1}} a^{-} \ldots \\
&\left(a^{+}\right)^{j_{2}-1-j_{1}} a^{-}\left(a^{+}\right)^{j_{1}-1} \phi .
\end{aligned}
$$

We observe first that if

$$
\left(a^{+}\right)^{2 n-j_{n}} a^{-}\left(a^{+}\right)^{j_{n}-1-j_{n-1}} a^{-} \cdots\left(a^{+}\right)^{j_{2}-1-j_{1}} a^{-}\left(a^{+}\right)^{j_{1}-1} \phi \neq 0
$$

then $j_{1}>1, j_{2}>3, \ldots, j_{n}>2 n-1$. Indeed if there exists $k \in\{1,2, \ldots, n\}$, such that $j_{k} \leq 2 k-1$, then there are at most $2 k-2$ epsilons before $\epsilon_{j_{k}}=-$, out of which $k-1$ are negative, namely $\epsilon_{j_{1}}, \epsilon_{j_{2}}, \ldots, \epsilon_{j_{k-1}}$, and therefore, there are at most $k-1$ positive epsilons left before $\epsilon_{j_{k}}$. Thus $a^{\epsilon_{j_{k}}} \cdots a^{\epsilon_{2}} a^{\epsilon_{1}} \phi=0$, since we start out from the vacuum space $G_{0}$ and do more steps backward $(k)$ than we do forward (at most $k-1$ ). This is the reason why the factors $\left(j_{1}-1\right)^{+},\left(j_{2}-3\right)^{+}, \ldots,\left(j_{n}-(2 n-1)\right)^{+}$and not $j_{1}-1, j_{2}-3, \ldots, j_{n}-(2 n-1)$ will appear later in this proof.

Claim 1: For all $k \in \mathbb{N}$, we have:

$$
\left[a^{-},\left(a^{+}\right)^{k}\right]=k\left(a^{+}\right)^{k-1}
$$

Indeed, this can be checked by induction on $k$. Since $\left[a^{-}, a^{+}\right]=I$, formula (10) holds for $k=1$. Let us assume that it holds for $k$ and prove that it 
also holds for $k+1$. This is true since:

$$
\begin{aligned}
a^{-}\left(a^{+}\right)^{k+1} & =\left(a^{-}\left(a^{+}\right)^{k}\right) a^{+} \\
& =\left(\left(a^{+}\right)^{k} a^{-}+\left[a^{-},\left(a^{+}\right)^{k}\right]\right) a^{+} \\
& =\left(\left(a^{+}\right)^{k} a^{-}+k\left(a^{+}\right)^{k-1}\right) a^{+} \\
& =\left(a^{+}\right)^{k}\left(a^{-} a^{+}\right)+k\left(a^{+}\right)^{k} \\
& =\left(a^{+}\right)^{k}\left(a^{+} a^{-}+\left[a^{-}, a^{+}\right]\right)+k\left(a^{+}\right)^{k} \\
& =\left(a^{+}\right)^{k}\left(a^{+} a^{-}+I\right)+k\left(a^{+}\right)^{k} \\
& =\left(a^{+}\right)^{k+1} a^{-}+\left(a^{+}\right)^{k}+k\left(a^{+}\right)^{k} \\
& =\left(a^{+}\right)^{k+1} a^{-}+(k+1)\left(a^{+}\right)^{k} .
\end{aligned}
$$

Thus we can see that $a^{-}\left(a^{+}\right)^{k+1}=\left(a^{+}\right)^{k+1} a^{-}+(k+1)\left(a^{+}\right)^{k}$ and subtracting $\left(a^{+}\right)^{k+1} a^{-}$from both sides of this equality we obtain that the commutator of $a^{-}$and $\left(a^{+}\right)^{k+1}$ is $\left[a^{-},\left(a^{+}\right)^{k+1}\right]=(k+1)\left(a^{+}\right)^{k}$. Thus according to the principle of mathematical induction, formula (10) holds for all positive integers $k$.

Let us come back to the general term of the sum from relation (9):

$$
\left(a^{+}\right)^{2 n-j_{n}} a^{-}\left(a^{+}\right)^{j_{n}-1-j_{n-1}} a^{-} \cdots\left(a^{+}\right)^{j_{2}-1-j_{1}} a^{-}\left(a^{+}\right)^{j_{1}-1} \phi
$$

and interchange the first two operators from the right $a^{-}$and $\left(a^{+}\right)^{j_{1}-1}$. If $j_{1}-1>0$, then using formula $(10)$ we get:

$$
\begin{aligned}
& \left(a^{+}\right)^{2 n-j_{n}} a^{-}\left(a^{+}\right)^{j_{n}-1-j_{n-1}} a^{-} \cdots\left(a^{+}\right)^{j_{2}-1-j_{1}} a^{-}\left(a^{+}\right)^{j_{1}-1} \phi \\
= & \left(a^{+}\right)^{2 n-j_{n}} a^{-} \cdots\left(a^{+}\right)^{j_{2}-1-j_{1}}\left(\left(a^{+}\right)^{j_{1}-1} a^{-}+\left[a^{-},\left(a^{+}\right)^{j_{1}-1}\right]\right) \phi \\
= & \left(a^{+}\right)^{2 n-j_{n}} a^{-} \cdots\left(a^{+}\right)^{j_{2}-1-j_{1}}\left(\left(a^{+}\right)^{j_{1}-1} a^{-}+\left(j_{1}-1\right)\left(a^{+}\right)^{j_{1}-2}\right) \phi \\
= & \left(a^{+}\right)^{2 n-j_{n}} a^{-}\left(a^{+}\right)^{j_{n}-1-j_{n-1}} a^{-} \cdots\left(a^{+}\right)^{j_{2}-1-j_{1}}\left(a^{+}\right)^{j_{1}-1} a^{-} \phi \\
+ & \left(j_{1}-1\right)\left(a^{+}\right)^{2 n-j_{n}} a^{-}\left(a^{+}\right)^{j_{n}-1-j_{n-1}} a^{-} \cdots\left(a^{+}\right)^{j_{2}-1-j_{1}}\left(a^{+}\right)^{j_{1}-2} \phi .
\end{aligned}
$$

Since $\phi \in G_{0}, a^{-}: G_{0} \rightarrow G_{-1}$, and $G_{-1}=\{0\}$, we conclude that $a^{-} \phi=0$. Thus from the last formula we obtain:

$$
\begin{aligned}
& \left(a^{+}\right)^{2 n-j_{n}} a^{-}\left(a^{+}\right)^{j_{n}-1-j_{n-1}} a^{-} \cdots\left(a^{+}\right)^{j_{2}-1-j_{1}} a^{-}\left(a^{+}\right)^{j_{1}-1} \phi \\
= & \left(j_{1}-1\right)\left(a^{+}\right)^{2 n-j_{n}} a^{-}\left(a^{+}\right)^{j_{n}-1-j_{n-1}} a^{-} \cdots\left(a^{+}\right)^{j_{2}-1-j_{1}}\left(a^{+}\right)^{j_{1}-2} \phi \\
= & \left(j_{1}-1\right)\left(a^{+}\right)^{2 n-j_{n}} a^{-}\left(a^{+}\right)^{j_{n}-1-j_{n-1}} a^{-} \cdots a^{-}\left(a^{+}\right)^{j_{2}-3} \phi,
\end{aligned}
$$

if $j_{1}>1$. Interchanging now $a^{-}$and $\left(a^{+}\right)^{j_{2}-3}$, if $j_{2}>3$, we obtain as before:

$$
\begin{aligned}
& \left(a^{+}\right)^{2 n-j_{n}} a^{-}\left(a^{+}\right)^{j_{n}-1-j_{n-1}} a^{-} \cdots\left(a^{+}\right)^{j_{2}-1-j_{1}} a^{-}\left(a^{+}\right)^{j_{1}-1} \phi \\
= & \left(j_{1}-1\right)\left(j_{2}-3\right)\left(a^{+}\right)^{2 n-j_{n}} a^{-}\left(a^{+}\right)^{j_{n}-1-j_{n-1}} a^{-} \cdots a^{-}\left(a^{+}\right)^{j_{3}-5} \phi .
\end{aligned}
$$


We now interchange $a^{-}$and $\left(a^{+}\right)^{j_{3}-5}$, if $j_{3}>5$, and so on until all the $a^{-}$ operators disappear. Each time we interchange $a^{-}$with a power of $a^{+}$, the power of $a^{+}$decreases by 1 , as we can see from formula (10). Because the total power of $a^{+}$was initially $n$ ( $n$ forward steps), we can see that after $n$ interchanges all the $a^{+}$operators disappear, too. Since $\phi=1$, we obtain in the end:

$$
\begin{aligned}
& \left(a^{+}\right)^{2 n-j_{n}} a^{-}\left(a^{+}\right)^{j_{n}-1-j_{n-1}} a^{-} \cdots\left(a^{+}\right)^{j_{2}-1-j_{1}} a^{-}\left(a^{+}\right)^{j_{1}-1} \phi \\
= & \left(j_{1}-1\right)\left(j_{2}-3\right) \cdots\left(j_{n}-(2 n-1)\right) \phi \\
= & \left(j_{1}-1\right)\left(j_{2}-3\right) \cdots\left(j_{n}-(2 n-1)\right),
\end{aligned}
$$

if $j_{1}>1, j_{2}>3, \ldots$, and $j_{n}>2 n-1$. If at least one $j_{k}$ is less than or equal to $2 k-1$, for $1 \leq k \leq n$, then

$$
\left(a^{+}\right)^{2 n-j_{n}} a^{-}\left(a^{+}\right)^{j_{n}-1-j_{n-1}} a^{-} \cdots\left(a^{+}\right)^{j_{2}-1-j_{1}} a^{-}\left(a^{+}\right)^{j_{1}-1} \phi=0,
$$

as was explained before. Thus we can say that, for all $1 \leq j_{1}<j_{2}<\cdots<$ $j_{n} \leq 2 n$, we have:

$$
\begin{aligned}
& \left(a^{+}\right)^{2 n-j_{n}} a^{-}\left(a^{+}\right)^{j_{n}-1-j_{n-1}} a^{-} \cdots\left(a^{+}\right)^{j_{2}-1-j_{1}} a^{-}\left(a^{+}\right)^{j_{1}-1} \phi \\
= & \left(j_{1}-1\right)^{+}\left(j_{2}-3\right)^{+} \cdots\left(j_{n}-(2 n-1)\right)^{+} .
\end{aligned}
$$

Applying now formulas (9) and (11) we obtain

$$
E\left[x^{2 n}\right]=\sum_{1 \leq j_{1}<j_{2}<\cdots<j_{n} \leq 2 n}\left(j_{1}-1\right)^{+}\left(j_{2}-3\right)^{+} \ldots\left(j_{n}-(2 n-1)\right)^{+} .
$$

Using now Lemma 2.1, we conclude that

$$
E\left[x^{2 n}\right]=(2 n-1) ! !
$$

for all positive integers $n$. This formula holds also for $n=0$, if we define $(-1) ! !:=1$.

Therefore we can say that, for all non-negative integers $i_{1}, i_{2}, \ldots, i_{d}$, $E\left[x_{1}^{i_{1}} x_{2}^{i_{2}} \cdots x_{d}^{i_{d}}\right]=\left(i_{1}-1\right) ! !\left(i_{2}-1\right) ! ! \cdots\left(i_{d}-1\right) ! !$, if $i_{1}, i_{2}, \ldots, i_{d}$ are all even numbers, and $E\left[x_{1}^{i_{1}} x_{2}^{i_{2}} \cdots x_{d}^{i_{d}}\right]=0$, otherwise. Thus $\mu$ has the same mixed moments as the standard Gaussian probability measure on $\mathbb{R}^{d}$. Since there is only one probability measure on $\mathbb{R}^{d}$ having the same moments as the standard Gaussian probability measure (namely the standard Gaussian measure itself), we conclude that $\mu$ must be the standard Gaussian measure. 


\section{Final Comments}

1) The above characterization of the standard Gaussian probability measure is just a particular case of a theorem that states that if $\mu$ and $\nu$ are two probability measures on $\mathbb{R}^{d}$, having finite moments of all orders, such that they have the same neutral (preservation) operators: $a_{\mu}^{0}(i)=a_{\nu}^{0}(i)$, and the same commutators between the annihilation and creation operators: $\left[a_{\mu}^{-}(j), a_{\mu}^{+}(k)\right]=\left[a_{\nu}^{-}(j), a_{\nu}^{+}(k)\right]$, for all $i, j, k \in\{1,2, \ldots, d\}$, then $\mu$ and $\nu$ have the same moments of all orders: $E_{\mu}\left[x_{1}^{i_{1}} x_{2}^{i_{2}} \cdots x_{d}^{i_{d}}\right]=E_{\nu}\left[x_{1}^{i_{1}} x_{2}^{i_{2}} \cdots x_{d}^{i_{d}}\right]$, for all non-negative integers $i_{1}, i_{2}, \ldots, i_{d}$. We believe that this theorem is a fundamental theorem of the theory of Interacting Fock Spaces and we will publish it in a forthcoming paper.

2) We have proven Lemma 2.1 by counting, the number of partitions of $2 n$ different people into $n$ teams, in two different ways. From the proof of Theorem 3.1, we can see that the second way of counting the teams is strongly connected with the fact that, for the standard Gaussian probability measure on $\mathbb{R}, a^{0}=0$ and $\left[a^{-}, a^{+}\right]=I$. How about the first way of counting the number of teams? The first way uses the multinomial coefficient $\left(\begin{array}{c}2 n \\ 2,2, \ldots, 2\end{array}\right)$. This way is also strongly connected to the Gaussian random variables if one recalls the Wick formula for calculating the expectation of a product of the form $\xi_{1} \cdot \xi_{2} \cdots \xi_{m}$, where $\xi_{1}, \xi_{2}, \ldots, \xi_{m}$ is a finite sequence of jointly normally distributed random variables with mean zero. This expectation is computed in terms of the complete Feynman diagrams. Namely, a complete Feynman diagram of order $m$ is a graph composed of $m$ vertices and some edges connecting these vertices, such that each vertex is connected to exactly one other vertex (no vertex is connected to itself, no vertex is connected to two different vertices, and no vertex is left unconnected to some other vertex). We place $\xi_{1}$ on one vertex, $\xi_{2}$ on another vertex, and so on $\xi_{m}$ on the last vertex. If we choose a complete Feynman diagram $\gamma$, in which $\xi_{1}$ is connected to $\xi_{i_{1}}, \xi_{2}$ to $\xi_{i_{2}}$, and so on $\xi_{n}$ to $\xi_{i_{n}}$, then we define:

$$
v(\gamma):=E\left[\xi_{1} \xi_{i_{1}}\right] E\left[\xi_{2} \xi_{i_{2}}\right] \ldots E\left[\xi_{n} \xi_{i_{n}}\right],
$$

where $E$ denotes the expectation. If we denote by $\Gamma(m)$ the set of all complete Feynman diagrams of order $m$ that can be obtained using $\xi_{1}, \xi_{2}$, $\ldots, \xi_{m}$ as vertices, then the following formula (called Wick formula) holds:

$$
E\left[\xi_{1} \xi_{2} \cdots \xi_{m}\right]=\sum_{\gamma \in \Gamma(m)} v(\gamma)
$$


See [6] for a proof. In particular, if $\xi_{1}=\xi_{2}=\cdots=\xi_{m}=X$, where $X$ is a standard Gaussian random variable, then we can see that there are no complete Feynman diagrams of order $m$, if $m$ is odd, while if $m=2 n$ is even, there are exactly $\left(\begin{array}{c}2 n \\ 2,2, \ldots, 2\end{array}\right) / n !=(2 n-1) ! !$ complete Feynman diagrams $\gamma$ made from $\xi_{1}, \xi_{2}, \ldots, \xi_{2 n}$, and for each of them $v(\gamma)=1$. Therefore, if $m$ is odd, $E\left[X^{m}\right]=0$, while if $m$ is even, the Wick formula yields:

$$
E\left[X^{m}\right]=\sum_{\gamma=1}^{(m-1) ! !} 1=(m-1) ! !
$$

In this way we can see the strong connection between the standard Gaussian probability measure and each of the sides of the combinatorial identity from Lemma 2.1.

\section{References}

1. Accardi, L. and Bożejko, M.: Interracting Fock space and Gaussianization of probability measures, Infinite Dimensional Analysis, Quantum Probability and Related Topics 1, (1998), 663-670.

2. Accardi, L., Kuo, H.-H., and Stan, A.: Characterization of probability measures through the cannonically associated Interacting Fock Spaces, Infinite Dimensional Analysis, Quantum Probability and Related Topics 7, (2004), 485-505.

3. Accardi, L., Lu, Y.G., and Volovich, I.: The QED Hilbert module and interacting Fock spaces; IIAS Reports No. 1997-008 (1997) International Institute for Advanced Studies, Kyoto.

4. Accardi, L. and Nahni, M.: Interacting Fock spaces and orthogonal polynomials in several variables; in "Non-Commutativity, Infinite-Dimensionality and Probability at the Crossroads", N. Obata et al. (eds.), World Scientific (2002), 192-205.

5. Chihara, T.S.: An Introduction to Orthogonal Polynomials. Gordon and Breach, 1978.

6. Janson, S.: Gaussian Hilbert Spaces. Cambridge Tracts in Math. 129, Cambridge University Press, 1997.

7. Kuo, H.-H.: White Noise Distribution Theory. CRC Press, Boca Raton, FL, 1996.

8. Obata, N.: White Noise Calculus and Fock Space. Lecture Notes in Mathematics 1571, Springer-Verlag, New York/Berlin, 1994.

9. Parthasarathy, K.R.: An Introduction to Quantum Stochastic Calculus. Birkhauser, 1992.

10. Szegö, M.: Orthogonal Polynomials, Coll. Publ. 23, Amer. Math. Soc., 1975.

11. Wiener, N.: The homogeneous chaos, American J. Math. 60 (1938), 897-936. 